\title{
РЕШЕНИЕ 23-ОЙ ПРОБЛЕМЫ ГИЛЬБЕРТА
}

П.М. Мазуркин, доктор технических наук, профессор, заведующий кафедрой природообустройства,

академик РАЕ и РАЕН, член Европейской Академии Естествознания ФГБОУ «Поволжский государственный технологический университет», Йошкар-Ола (Россия), kaf_po@mail.ru

1. Постановка задачи. Проблема развития методов вариационного исчисления (23-я проблема Гильберта) до сих пор не разрешена, хотя в этом направлении много было сделано самим Гильбертом [1]. Математическую деятельность Давида Гильберта условно можно разделить на две разнонаправленные концепции (векторы понимания): а) интегральные уравнения и физика (в общем смысле, включая все науки: астрономия, биология и экология, эконометрика, технология и др.); б) алгебраические числовые поля и инварианты. Два этих вектора понимания объединяются в теории аксиоматики Гильберта.

Таким образом, аксиоматика лежит в основе обоих подходов - от математика к физику-теоретику (первый вектор) и, наоборот, от экспериментатора к математику. Монарный подход математика-теоретика к развитию абстрактной математики мы не рассматриваем, так как на многих примерах (более 100 тысяч) статистического (вероятностного) моделирования убедились в том, что решение 23-й проблемы Гильберта находится не в математике или физике по отдельности, а именно в переходе от экспериментальной физики (измерения в физике, астрономии, биологии и экологии, социологии и эконометрике, технике и технологии) к методам прикладной математики.

Ключом к пониманию классическими математиками предлагаемой нами методологии идентификации инвариантов по реальным алгебраическим числовым полям, как результатам многофакторных физических измерений, является эвристическое утверждение: «Руководящим принципом для Гильберта служила аналогия с соответствующими проблемами для алгебраических функций от одной переменной» [1]. Но у нас инвариантами являются гладкие и нелинейные устойчивые законы, включая и волновые функции, применимые к вещественным числовым полям (статистическим выборкам). Каждый физический фактор обособленно может приниматься за объясняющую переменную.

2. Методология идентификации закономерностей. Подробнее отдельные особенности предложенной методологии даны в статье [2]. Технология пользования программной средой CurveExpert показана в пособии [9].

Идентификация по числовому полю или табличной модели имеет этапы:

1) эвристическая идентификация сути изучаемого явления или процесса по результатам измерений и анализа табличной модели (априорная информация) и осмысления в ходе выявления конструкции у однофакторной закономерности по её аддитивным составляющим (апостериорная информация);

2) структурная идентификация каждой однофакторной математической (статистической) функции как суммы инвариантов в виде асимметричных 
вейвлет-сигналов с переменными амплитудой и периодом колебания;

3) параметрическая идентификация наращиваемой по отдельным инвариантам структуры устойчивой статистической закономерности в программной среде типа CurveExpert (http://www.curveexpert.net).

2.1. Идентификация эвристическая. Она предполагает знание предмета исследования, поэтому здесь важен переход от физики к математике, а не наоборот. Главное эвристическое значение приобретает заданное числовое поле, как правило, оформляемое по результатам измерений в виде таблицы. В ней неявно заключен весь содержательный смысл (эвристика) и понимание (концепция) автора измерений, то есть сущностный смысл эксперимента.

Мы давно отказались от методологии аппроксимации и, соответственно, вообще не применяем программы статистики из Excel. В идентификации первый этап (вариация функций) из теории аппроксимации исключается, - устойчивые законы заранее заданы как инварианты. Из них как из кирпичиков можно создавать различные математические конструкты. Использование в каждом конкретном примере статистического (вероятностного) моделирования того или иного устойчивого закона (по таблице 1 их всего семь) требует предварительного эвристического осмысления содержания задачи моделирования.

Таким образом, на эвристическом уровне 23-я проблема Гильберта нами пока недостаточно формализована. Но проблема развития методов вариационного исчисления была решена на структурном и параметрическом уровнях анализа и синтеза. Вариация функций сводится к осознанному отбору устойчивых законов и конструированию на их основе волновых закономерностей.

2.2. Идентификация структурная. Декарт предполагал существование одного-единственного алгебраического уравнения, пригодного как вариант решения для любых типов интегральных уравнений. Гильберт мечтал об инвариантах, из них как из кирпичиков будет собираться универсальное уравнение.

Наши универсальные инварианты даны в таблице 1.

Таблица 1 - Математические конструкты (исходные инварианты или кирпичики Гильберта) для составления статистической модели

\begin{tabular}{|c|c|}
\hline $\begin{array}{c}\text { Фрагменты без предыстории } \\
\text { изучаемого явления или процесса }\end{array}$ & $\begin{array}{c}\text { Фрагменты с предысторией } \\
\text { изучаемого явления или процесса }\end{array}$ \\
\hline $\begin{array}{l}y=a x-\text { закон линейного роста или спада } \\
\text { (при отрицательном знаке перед правой сто- } \\
\text { роной формулы линейного изменения) }\end{array}$ & $\begin{array}{l}y=a-\text { закон не влияния принятой объяс- } \\
\text { няющей переменной на показатель, который } \\
\text { имеет собственную предысторию значений }\end{array}$ \\
\hline $\begin{array}{l}y=a x^{b} \text { - закон показательного роста (закон } \\
\text { показательной гибели } y=a x^{-b} \text { не является } \\
\text { устойчивым, из-за бесконечности значения } \\
\text { показателя при нулевом значении объяс- } \\
\text { няющей переменной) }\end{array}$ & $\begin{array}{l}y=a \exp ( \pm c x) \text { - закон Лапласа в математике } \\
\text { (Ципфа в биологии, Парето в экономике, } \\
\text { Мандельброта в физике) экспоненциального } \\
\text { роста или гибели, относительно которого } \\
\text { Лаплас создал методологию операторного } \\
\text { исчисления }\end{array}$ \\
\hline $\begin{array}{l}y=a x^{b} \exp (-c x) \text { - биотехнический закон в } \\
\text { упрощенной форме (П.М. Мазуркин), когда } \\
\text { показательный рост постепенно получает } \\
\text { экспоненциальное торможение }\end{array}$ & $\begin{array}{l}y=a \exp \left( \pm c x^{d}\right) \text { - закон экспоненциального } \\
\text { роста или гибели в полной форме (конструк- } \\
\text { ции), который имеет интенсивность, не рав- } \\
\text { ную единице (П.М. Мазуркин) }\end{array}$ \\
\hline \multicolumn{2}{|c|}{$y=a x^{b} \exp \left(-c x^{d}\right)$ - биотехнический закон, общий для шести конструктов (П.М. Мазуркин) } \\
\hline
\end{tabular}


Они сгруппированы по принципу «от простого к сложному». По сути, фрагменты и сам биотехнический закон являются «кирпичиками Гильберта» для построения, в ходе процесса структурно-параметрической идентификации, аддитивной конструкции статистической модели с трендами и вейвлетами.

Инварианты колебательных возмущений в виде асимметричных вейвлетсигналов также включают в себя конструкты из таблицы 1 как амплитуда (половина) и полупериод.

В таблице 1 показаны наиболее встречающиеся инварианты (фрагменты). У них впереди могут быть расположены оперативные константы «+» или «-». Шесть устойчивых законов распределения являются частными случаями биотехнического закона, показанного внизу таблицы 1. В названии закона слово «биотехнический» означает, что мы придерживаемся идей В.И. Вернадского о космической функции жизни. Это доказывается в ${ }^{4}$ тем, что ряд Фибоначчи является неким «родственником» ряда положительных простых чисел [4].

Если известны эвристическая предыстория формирования числового поля (табличной модели), то вполне возможна смысловая расшифровка каждого вейвлет-сигнала, у которого вейвлет (волновая функция) в своей конструкции содержит те или иные математические инварианты из таблицы 1 [5-8].

Устойчивые законы и закономерности на их основе делают выбор уравнения для последующей идентификации на статистических данных (числовых полях) вполне осмысленным, и поэтому вероятностное моделирование остается только при случайном поиске программной средой типа CurveExpert значений параметров у искомой модели. Поэтому из теории идентификации первый этап (выбор случайной структуры уравнения) исключается и остается только второй этап - случайная идентификация значений параметров модели.

Структура модели идентифицируется обработкой исходных данных так:

- вначале выявить детерминированные нелинейные закономерности;

- затем дополнять эти тренды колебательными возмущениями.

2.3. Идентификация параметрическая. Она выполнялась в программной среде CurveExpert-1.40 (http://www.curveexpert.net) и информационной технологией идентификации пользуются студенты (будущие бакалавры и магистры), а также аспиранты и докторанты. На сквозных примерах методология структурно-параметрической идентификации подробно показана в книге [4].

Выбор структуры искомой модели, которая является алгебраическим решением по Декарту для неизвестной первообразной по волновым уравнениям, имеющим переменные амплитуду и полупериод (половины частоты как обратная величина полупериода) колебательного возмущения объекта исследования, выполняется из устойчивых законов (инвариантов), приведенных в таблице 1.

Процесс параметрической идентификации автоматически прекращается по условию достижения параметров модели некоторого минимального приращения и останавливается при достижении моделью погрешности измерений.

3. Частные случаи биотехнического закона. Все известные гладкие законы распределения являются частными случаями биотехнического закона:

1) $y=a_{1}$ при $a_{2}=0, a_{3}=0$ - отсутствие влияния переменной на показатель; 
2) $y=x$ при $a_{1}=1, a_{2}=1, a_{3}=0$ - тождество, переменная есть показатель;

3) $y=a_{1} x-$ пропорциональное влияние $x$ на $y$ при $a_{2}=1, a_{3}=0$;

4) $y=x \exp (-x)$ - идеальный биотехнический закон, $a_{1}=1, a_{2}=1, a_{3}=1, a_{4}=1$;

5) $y=a_{1} x \exp (-x)=1$ - мальтузианский рост с торможением $a_{2}=1, a_{3}=1, a_{4}=1$;

6) $y=a_{1} x \exp \left(-a_{3} x\right)$ - распределение Релея при $a_{2}=1, a_{4}=1$;

7) $y=a_{1} x^{a_{2}}$ - аллометрический рост при $a_{3}=0$;

8) $y=a_{1} x^{-a_{2}}-$ показательный закон Ципфа-Парето-Мандельброта, $a_{3}=0$;

9) $y=a_{1} \exp \left(-a_{3} x\right)$ - при $a_{2}=0, a_{4}=1$ экспоненциальный закон ЦипфаПарето-Мандельброта;

10) $y=a_{1} \exp \left(-a_{3}(x-\bar{x})^{2}\right)-$ закон нормального распределения Гаусса при $a_{2}=0, a_{4}=2$, где $\bar{x}$-среднее значение (математическое ожидание) переменной $x$; здесь $a_{1}=1 /(\sigma \sqrt{2 \pi}), a_{2}=1 /\left(2 \sigma^{2}\right)$, где $\sigma$-отклонение среднее квадратичное;

11) $y=a_{1} x \exp \left(-a_{3} x^{2}\right)$ - распределение Релея при $a_{2}=1, a_{4}=2$;

12) $y=a_{1}\left(1+a_{5} \exp \left(-a_{3} x\right)\right)^{-1}$ - закон Перла при $a_{2}=0, a_{4}=1$ и дополнении $a_{5}$ к математическому конструкту силы противодействия других факторов;

13) $y=a_{1}\left(a_{5}+\exp \left(a_{6} \exp \left(-a_{3} x\right)\right)\right)^{-1}-$ закон А.Ф.Каменева на основе усложнения силы противодействия;

14) $y=a_{0}+a_{1} x^{a_{2}}-$ закон Никитина для хода роста древостоев при $a_{3}=0$;

15) $y=a_{1}\left(1-\exp \left(-a_{3} x\right)\right)^{a_{5}}$ - закон Митчерлих I для хода роста древостоев, рекомендованный ВНИИЛМ как официально утвержденной регрессионной модели для лесоводов и таксаторов;

16) $y=x^{a_{2}}\left(a_{5}+a_{6} x^{a_{2}-1}+a_{7} x^{a_{2}}\right)^{-1}-$ закон Йошида II для хода роста древостоев, при $a_{1}=1$ и замене функции противодействия на трехчленную формулу; этот закон наиболее точен для некоторых лесоводственных условий, однако он труднообъясним содержательно;

17) $y=\pi a_{1}\left(a_{1}^{2}+(x-b)^{2}\right)^{-1}$ - распределение Коши при $a_{2}=0$ и иной форме силы противодействия, где $b$ - параметр, определяющий одновременно значение моды и медианы;

18) $y=a_{1} \exp (-x) \exp \left(-a_{3}(x-\bar{x})^{2}\right)$ - распределение логнормальное, когда значение $x$ в силе действия заменено на $\exp (x)$, причем $a_{2}=-1$;

19) $y=a_{1} x^{2} \exp \left(-a_{3} x^{2}\right)$ - распределение Максвелла при $a_{2}=2, a_{4}=2$, $a_{1}=(\sqrt{2 / \pi}) \alpha^{-3}, \quad a_{3}=1 /\left(2 \alpha^{2}\right)$, где $\alpha$ - параметр распределения; $\hat{\alpha}=0.6267 \bar{x}$;

20) $y=a_{1} \exp \left(-a_{3} x^{2}\right)$ - ряд Грама-Шарлье типа А, иногда используемый для изучения рядов распределения значений таксационных показателей множеств деревьев, причем $a_{1}=1 / \sqrt{2 \pi}, a_{3}=1 / 2, a_{2}=0, a_{4}=2$;

21) $y=a_{1} \exp \left(-a_{1} x\right)$ - распределение экспоненциальное, $a_{2}=0, a_{3}=a_{1}, a_{4}=1$;

22) $y=a_{1} x^{a_{2}} \exp \left(-a_{3} x\right)$ - распределение Эрланга при $a_{1}=(n \mu)^{n} / \Gamma(n)$, $a_{2}=n-1, a_{3}=n \mu, n \geq 1$ и $\mu>0$ - параметры распределения, Г(n) - гамма-функция;

23) $y(x=m)=a_{1} \lambda^{a_{2}} \exp (-\lambda)$ - распределение Пуассона при $a_{1}=m !, a_{2}=m$, $a_{3}=1, a_{4}=1$, где $m=0,1,2, \ldots$ целые неотрицательные числа; 
24) $y=a_{1} \exp \left(-a_{3} t^{2}\right)$ - распределение нормальное стандартизованное при $a_{1}=1 / \sqrt{2 \pi}, a_{2}=0, a_{3}=1 / 2, t=(x-\bar{x}) / \sigma$.

4. Уровни адекватности закономерностей. В таблице 2 приведены интервалы изменения коэффициента корреляции как меры адекватности модели.

Таблица 2 - Уровни адекватности статистических моделей

\begin{tabular}{|c|c|c|c|c|}
\hline \multirow[b]{2}{*}{$\begin{array}{c}\text { Интервал коэф- } \\
\text { фициента } \\
\text { корреляции }\end{array}$} & \multicolumn{4}{|c|}{ Характер тесноты связи между факторами } \\
\hline & $\begin{array}{l}\text { существующая } \\
\text { классификация }\end{array}$ & $\begin{array}{c}\text { шкала для } \\
\text { технических } \\
\text { измерений } \\
\end{array}$ & \begin{tabular}{|c|} 
шкала для \\
прецизионных \\
измерений
\end{tabular} & $\begin{array}{c}\text { шкала для генной } \\
\text { инженерии и рядов це- } \\
\text { лых простых чисел }\end{array}$ \\
\hline 1 & \multirow{6}{*}{ сильная связь } & однозначная & однозначная & однозначная \\
\hline $0.999 \ldots 1.0000$ & & \multirow{4}{*}{ сильнейшая } & \multirow{2}{*}{ почти однозначная } & почти однозначная \\
\hline $0,99 \ldots 1,000$ & & & & чрезвычайно сильная \\
\hline $0,95 \ldots 0,99$ & & & сверхсильная & сверхсильная \\
\hline $0,90 \ldots 0,95$ & & & сильнейшая & сильнейшая \\
\hline $0,7 \ldots 0,9$ & & сильная & сильная & сильная \\
\hline $0,5 \ldots 0,7$ & \multirow{2}{*}{ слабая связь } & средняя & средняя & средняя \\
\hline $0,3 \ldots 0,5$ & & слабоватая & слабоватая & слабоватая \\
\hline $0,1 \ldots 0,3$ & \multirow{3}{*}{ нет связи } & слабая & Слабая & слабая \\
\hline $0,0 \ldots 0,1$ & & слабейшая & слабейшая & слабейшая \\
\hline 0 & & нет связи & нет связи & нет связи \\
\hline
\end{tabular}

Существующая шкала квантификации тесноты связи между принятыми факторами (нет связи, слабая и сильная связь) является очень грубой.

Критерием (количественной мерой) идентификации становится погрешность измерений при составлении числового поля, т.е. таблицы исходные данных. Например, простые числа и их ряды [4] не имеют погрешности измерения: они абсолютно достоверны, добротны и надежны. Другие виды исходных данных имеют разную погрешность. Таким образом, математические числовые объекты - наилучшие для идентификации законов-инвариантов.

На втором месте находятся прецизионные измерения в астрономии, физике, технике и технологии, других областях науки. На третье место встают биологические объекты (у нас - почва [6] и деревья [7]), и их поведение имеет четко проявляющийся колебательный характер, поэтому линейные и линеаризованные модели биологам вообще не подходят. Самые высокие погрешности имеют социально-экономические измерения $[5,8]$ из-за их субъективности.

Нами было доказано, что именно анализ слабых связей, отбрасываемых при линейном моделировании, и применении среднеарифметической факторной связи по закону Гаусса (нормального распределения), дает новые научные идеи и последующие научно-технические решения (за 10 лет с участием студентов было получено более 95 патентов на изобретения РФ).

Анализ литературы по математической статистике показал, что в аппроксимации сплошь и рядом применяется линейная модель или же не имеющий физического смысла алгебраический полином. Это происходит из-за того, что линейная модель инвариантна к любому типу распределения и отрицательным значениям объясняющей переменной, в том числе и явно негауссовой структуры, и даже к скедастическим данным измерений с переменной дисперсией. 
Нами предложена для технических экспериментов, в которых погрешность измерений не превышает $5 \%$, другая шкала (третий столбец таблицы 2). Однако выяснилось, что этой шкалы уровней адекватности также недостаточно.

Для многих природных (биологических) объектов и результатов прецизионных физических измерений пришлось ввести еще два интервала уровня адекватности по четвертому столбцу таблицы 1, что нами было выполнено только при моделировании распределений рядов простых чисел [1]. А для генной инженерии, по данным статьи (Jayodita C Sanghvi1 et al. Accelerated discovery via a whole-cell model. Nat. Methods. Published online 3 November 2013 doi:10.1038/nmeth.2724), пришлось ввести еще один уровень адекватности, который нами был открыт только для рядов целых простых чисел.

5. Концепция моделирования по статистическим выборкам. Статистическая выборка - это многофакторное числовое поле в виде табличной модели. Этим определением она отличается от таблиц статистических изысканий. Причем необязательно все клетки таблицы должны быть заполненными.

При этом табличная модель необязательно имеет эвристические пояснения. Как правило, авторы измерений, приводя в своих публикациях таблицы данных, дают неверные содержательные толкования. Этот феномен эвристической формализации связан с тем, что таблица результатов измерений, даже если она составлена авторами правильно, не может быть содержательно осмыслена без проведения факторного анализа [3, 6, 8] с математическим моделированием связей между парами факторов для выявления бинарных связей.

Тогда первичной становится табличная модель (исходное числовое поле), которая оценивается по погрешности проведенных измерений, а вторичным является искомое сложное алгебраическое уравнение (в смысле Декарта), составленное из инвариантов таблицы 1 (в смысле кирпичиков Гильберта). Этот процесс есть статистическая идентификация. Сама первообразная в виде неизвестного интегрального уравнения становится не нужным, хотя, может быть, кто-то и сумеет получать интегралы по нашим моделям.

Это - великое созидание, как уравнения Максвелла в электромагнетизме.

6. Детерминированная модель. В общем случае не волновая модель (тренд) содержит сумму двух биотехнических законов в виде уравнения

$$
y_{m}=y_{m 1}+y_{m 2}, y_{m 1}=a_{1} x^{a_{2}} \exp \left(-a_{3} x^{a_{4}}\right), y_{m 2}=a_{5} x^{a_{6}} \exp \left(-a_{7} x^{a_{8}}\right),
$$

где $y_{m}$ - тренд, $x$ - объясняющая переменная, $a_{1} \ldots a_{8}$ - параметры модели (1).

При этом каждый параметр модели (1) имеет физический смысл [9].

Не волновой характер общая модель (1) получает в двух случаях:

1) когда шаг дискретности измерений слишком большой по сравнению с периодом колебательного возмущения измеряемого реального процесса (например, импульс электрокардиограммы требует регистрации через $0,001 \mathrm{c}$ );

2) когда интервал процесса измерений мал по сравнению с полупериодом колебательного возмущения измеряемого показателя (например, среднегодовая температура в точке Земли требует регистрации за 1000 лет и более).

7. Асимметричный вейвлет. Мы придерживаемся концепции Декарта о необходимости применении алгебраического уравнения общего вида напрямую 
как конечного математического решения неизвестных интегральных уравнений. Для обобщения был предложен новый класс волновых функций [2-9].

Условиям существования в реальной действительности наиболее полно удовлетворяет обобщённая асимметричная вейвлет-функция вида

$$
y=\sum_{i=1}^{m} y_{i}, \quad y_{i}=a_{1 i} x^{a_{2 i}} \exp \left(-a_{3 i} x^{a_{4 i}}\right) \cos \left(\pi x /\left(a_{5 i}+a_{6 i} x^{a_{7 i}} \exp \left(-a_{8 i} x^{a_{9 i}}\right)-a_{10 i}\right),\right.
$$

где у - показатель (зависимый фактор), i - номер составляющей (2), m - количество членов в модели (2), причем косинус является связующим звеном между геометрией и алгеброй, х - объясняющая переменная (влияющий фактор), $a_{1} \ldots a_{10}$ - параметры, принимающие числовые значения в ходе структурнопараметрической идентификации математического конструкта (2).

В большинстве случаев для идентификации искомых закономерностей по известным табличным моделям достаточна усеченная конструкция (по формуле частоты колебания) асимметричного вейвлета типа

$$
y=\sum_{i=1}^{m} y_{i}, y_{i}=a_{1 i} x^{a_{2 i}} \exp \left(-a_{3 i} x^{a_{4 i}}\right) \cos \left(\pi x /\left(a_{5 i}+a_{6 i} x^{a_{7 i}}\right)-a_{8 i}\right) .
$$

При этом количество $m$ членов в наших примерах достигала до 90 и более.

Как правило, общая стохастическая волновая функция (3), в которой не волновые части (1) становятся частными случаями и показывают детерминированное на интервале времени измерений поведение объекта исследования. Это позволяет идентифицировать составной статистической моделью поведение многих математических, астрономических, биологических и экологических, социально-экономических и иных объектов.

8. Динамический ряд как череда сигналов. Физико-математический подход предполагает понимание смысла динамического ряда как отражения какого-то составного процесса или же множества последовательно и параллельно происходящих природных и/или природно-антропогенных процессов.

Впервые удалось получать модели многих типов рядов динамики [3-9] на концепции аддитивного разложения любого динамического ряда на множество вейвлет-сигналов.

Сигнал - это материальный носитель информации. А информация нами понимается как мера взаимодействия. Сигнал может генерироваться, но его приём не обязателен. Так, например, ряд простых чисел известен несколько тысяч лет, но суть его как множества сигналов до сих пор не была раскрыта [4]. Сигналом может быть любой физический процесс или его часть. Получается, что изменение множества неизвестных сигналов давно известно, например, через ряды гидрометеорологических измерений во многих точках планеты. Однако до сих пор не получены их статистические модели.

Тогда любое уравнение типа (3) можем записать как вейвлет-сигнал вида

$$
\begin{array}{r}
y_{i}=A_{i} \cos \left(\pi x / p_{i}-a_{8 i}\right), \\
A_{i}=a_{1 i} x^{a_{2 i}} \exp \left(-a_{3 i} x^{a_{4 i}}\right), p_{i}=a_{5 i}+a_{6 i} x^{a_{7 i}},
\end{array}
$$

где $A_{i}$ - амплитуда (половина) вейвлета (ось $y$ ), $p_{i}$ - полупериод волны (ось $x$ ).

По формуле (4) с двумя фундаментальными физическими постоянными $e$ (число Непера или число времени) и $\pi$ (число Архимеда или число простран- 
ства) образуется изнутри изучаемого явления и/или процесса квантованный вейвлет-сигнал. Понятие асимметричного вейвлет-сигнала позволяет абстрагироваться от физического смысла самих динамических рядов (в общем случае не только динамических) и рассматривать их аддитивное разложение.

Выводы. Нами доказана возможность решения проблемы развития методов вариационного исчисления (23-я проблема Гильберта). Для этого были получены кирпичики Гильберта, позволяющие конструировать обобщенное алгебраическое уравнение по Декарту. Создана методология идентификации любых явлений и процессов, требующая только этапа параметрической идентификации суммы асимметричных вейвлет-сигналов. Однако программная среда типа CurveExpert для такой идентификации этого весьма трудоемкая и при этом ограничена по количеству одновременно идентифицируемых параметров модели. Поэтому по нашим сценариям нужно создать новую программную среду для её применения на суперкомпьютерах петафлопного класса.

\section{СПИСОК ЛИТЕРАТУРЫ}

1. Теория инвариантов. Алгебраические числовые поля. Аксиоматика. Интегральные уравнения. URL: http://www.ega-math.narod.ru/Reid/Weyl.htm.

2. Мазуркин П.М. Идентификация статистических устойчивых закономерностей // Наука и мир: международ. научный журнал. 2013. № 3(3). С. 28-33.

3. Мазуркин П.М. Вейвлет-анализ альфа-активности ${ }^{239} \mathrm{Pu}$ во времени солнечного затмения // Наука и мир: междунар. научный журнал. 2014. № 1(5). C.94-104.

4. Мазуркин П.М. Закономерности простых чисел. Germany: Palmarium Academic Publishing, 2012. 280 c.

5. Мазуркин П.М. Колебания инновационной деятельности в России // Фундаментальные исследования. № 10 (ч.11). 2013 С. 2508-2513.

6. Мазуркин П.М. Коррелятивная вариация: учеб. пос. с грифом УМО РАЕ. Йошкар-Ола: Поволжский ГТУ, 2013. 120 с.

7. Мазуркин П.М. Биокаркас территории: учеб. пос. с грифом УМО РАЕ. Йошкар-Ола: Поволжский ГТУ, 2013. 156 с.

8. Мазуркин П.М. Закономерности вейвлет-анализа показателей инновационной активности небольших и крупных стран // Международный журнал прикладных и фундаментальных исследований. № 3. 2013. С.96-102.

9. Мазуркин П.М., Филонов А.С. Математическое моделирование. Идентификация однофакторных статистических закономерностей: учеб. пос. Йошкар-Ола: МарГТУ, 2006. 292 с.

\section{SOLUTION OF THE TWENTY THIRD PROBLEM OF GILBERT (C)2014}

P.M. Mazurkin, Doctor of Technical Sciences, Professor, Academician of the Russian Academy of Natural Sciences and the Russian Academy of Natural Sciences, Member of the European Academy of Natural Sciences,

Volga State Technological University (Yoshkar-Ola), Russia, kaf po@mail.ru 